\title{
Research Article \\ On the Periods of 2-Step General Fibonacci Sequences in the Generalized Quaternion Groups
}

\author{
Bahram Ahmadi and Hossein Doostie \\ Department of Mathematics, Science and Research Branch, Islamic Azad University, \\ P.O. Box 14515-1775, Tehran 14778-93855, Iran \\ Correspondence should be addressed to Bahram Ahmadi, b.ahmadi.math@gmail.com
}

Received 12 October 2012; Accepted 3 December 2012

Academic Editor: Carlo Piccardi

Copyright (C 2012 B. Ahmadi and H. Doostie. This is an open access article distributed under the Creative Commons Attribution License, which permits unrestricted use, distribution, and reproduction in any medium, provided the original work is properly cited.

We study 2-step general Fibonacci sequences in the generalized quaternion groups $Q_{4 n}$. In cases where the sequences are proved to be simply periodic, we obtain the periods of 2-step general Fibonacci sequences.

\section{Introduction}

The study of the Fibonacci sequences in groups began with the earlier work of Wall [1] in 1960, where the ordinary Fibonacci sequences in cyclic groups were investigated. In the mideighties, Wilcox [2] extended the problem to the abelian groups. In 1990, Campbell et al. [3] expanded the theory to some classes of finite groups. In 1992, Knox proved that the periods of $k$-nacci ( $k$-step Fibonacci) sequences in the dihedral groups are equal to $2 k+2$, in [4]. In the progress of this study, the article [5] of Aydin and Smith proves that the lengths of the ordinary 2-step Fibonacci sequences are equal to the lengths of the ordinary 2-step Fibonacci recurrences in finite nilpotent groups of nilpotency class 4 and a prime exponent, in 1994.

Since 1994, the theory has been generalized and many authors had nice contributions in computations of recurrence sequences in groups and we may give here a brief of these attempts. In [6,7] the definition of the Fibonacci sequence has been generalized to the ordinary 3-step Fibonacci sequences in finite nilpotent groups. Then in [8] it is proved that the period of 2-step general Fibonacci sequence is equal to the length of the fundamental period of the 2-step general recurrence constructed by two generating elements of a group of nilpotency class 2 and exponent $p$. In [9] Karaduman and Yavuz showed that the periods of the 2-step Fibonacci recurrences in finite nilpotent groups of nilpotency class 5 and a prime exponent are $p \cdot k(p)$, for $2<p \leq 2927$, where $p$ is a prime and $k(p)$ is the period of the ordinary 
2-step Fibonacci sequence. The main role of $[10,11]$ in generalizing the theory was to study the 2-step general Fibonacci sequences in finite nilpotent groups of nilpotency class 4 and exponent $p$ and to the 2-step Fibonacci sequences in finite nilpotent groups of nilpotency class $n$ and exponent $p$, respectively.

One may consult $[12,13]$ to see the results of the Fibonacci sequences in the modular groups concerning the periodicity of 2-step Fibonacci sequences constructed by two generating elements.

Going on a detailed literature in this area of research, we have to mention certain essential computation on the Fibonacci lengths of new structures like the semidirect products, the direct products, and the automorphism groups of finite groups which have been studied in [14-19]. Finally, we refer to [20] where Karaduman and Aydin studied the periodicity property of 2-step general Fibonacci sequences in dihedral groups and the goal of this paper is to calculate the periods of 2-step general Fibonacci sequences in the generalized quaternion groups.

Let $G=\left\langle a_{0}, a_{1}, a_{2}, \ldots, a_{j-1}\right\rangle$ be a finite group. A $k$-nacci sequence in group $G$ is a sequence $\left\{x_{n}\right\}_{n=0}^{\infty}$ of group elements for which each element is defined by $x_{0}=a_{0}, x_{1}=$ $a_{1}, \ldots, x_{j-1}=a_{j-1}$,

$$
x_{n}= \begin{cases}x_{0} x_{1} \cdots x_{n-1}, & \text { for } j \leq n<k, \\ x_{n-k} x_{n-k+1} \cdots x_{n-1}, & \text { for } n \geq k .\end{cases}
$$

This sequence of the group $G$ is denoted by $F_{k}\left(G ; a_{0}, a_{1}, a_{2}, \ldots, a_{j-1}\right)$. We also call a 2-nacci sequence of group elements a Fibonacci sequence of a finite group. A finite group $G$ is $k$ nacci sequenceable if there exists a $k$-nacci sequence of $G$ such that every element of the group appears in the sequence. A sequence of group elements is periodic if after a certain point, it consists only of repetitions of a fixed subsequence. The number of elements in the repeating subsequence is called the period of the sequence. For example, the sequence $a, b, c, d, d, e, b, c, d, e, \ldots$ is periodic after the initial element $a$ and has period 4 . We denote the period of a $k$-nacci sequence $F_{k}\left(G ; a_{0}, a_{1}, a_{2}, \ldots, a_{j-1}\right)$ by $P_{k}\left(G ; a_{0}, a_{1}, a_{2}, \ldots, a_{j-1}\right)$. A sequence of group elements is called simply periodic with period $k$ if the first $k$ elements in the sequence

form a repeating subsequence. For example, the sequence $a, b, c, d, e, f, a, b, c, d, e, f, \ldots$ is simply periodic with period 6 . The following theorem is well known.

Theorem 1.1 (see [4]). Every $k$-nacci sequence in a finite group is simply periodic.

\section{Main Theorems}

The generalized quaternion group $Q_{4 n},(n \geq 2)$ is a group with a presentation of the form

$$
\left\langle a, b \mid a^{2 n}=1, a^{n}=b^{2}, b^{-1} a b=a^{-1}\right\rangle .
$$

It is easy to see that $Q_{4 n}$ is of order $4 n, a$ has order $2 n, b$ has order 4 , and the relation $a^{k} b=b a^{-k}$ holds for all $k \in \mathbb{Z}$.

First we consider the following lemma which will be used frequently without further reference. 
Lemma 2.1. For an integer $r$, where $1 \leq r<2 n$, and for $s \in\{1,3\}$, the following relations hold in $Q_{4 n}$ :

(i) $\left(a^{r} b^{s}\right)^{t}=b^{s t}$, where $t$ is any even integer;

(ii) $\left(a^{r} b^{s}\right)^{t}=a^{r} b^{\text {st }}$, where $t$ is any odd integer.

Proof. We may use an induction method on $t$ for both parts, simultaneously.

Theorem 2.2. $P_{k}\left(Q_{4 n} ; a, b\right)=2 k+2$.

Proof. Obviously, the order of $a b$ is 4 . If $k=2$, then

$$
\begin{gathered}
x_{0}=a, \quad x_{1}=b, \quad x_{2}=a b, \quad x_{3}=a^{n-1}, \\
x_{4}=a^{n+2} b, \quad x_{5}=a b, \quad x_{6}=a, \quad x_{7}=b, \ldots,
\end{gathered}
$$

and hence the sequence has period 6 . If $k=3$, then

$$
\begin{gathered}
x_{0}=a, \quad x_{1}=b, \quad x_{2}=a b, \quad x_{3}=(a b)^{2}=b^{2}, \quad x_{4}=a^{-1}, \\
x_{5}=a^{2} b^{3}, \quad x_{6}=a b, \quad x_{7}=1, \quad x_{8}=a, \quad x_{9}=b, \ldots,
\end{gathered}
$$

and so the period is 8 . Now let $k \geq 4$. Then, the first $k+1$ elements of $F_{k}\left(Q_{4 n} ; a, b\right)$ are

$$
x_{0}=a, \quad x_{1}=b, \quad x_{2}=a b, \quad x_{3}=(a b)^{2}, \quad x_{4}=(a b)^{4}, \ldots, x_{k}=(a b)^{2^{k-2}} .
$$

Since $a b$ is of order 4 , this sequence reduces to

$$
x_{0}=a, \quad x_{1}=b, \quad x_{2}=a b, \quad x_{3}=b^{2}, \quad x_{j}=1, \quad(4 \leq j \leq k) .
$$

Thus,

$$
\begin{aligned}
& x_{k+1}=\prod_{i=1}^{k} x_{i}=b(a b) b^{2}=a^{-1}, \\
& x_{k+2}=\prod_{i=2}^{k+1} x_{i}=(a b) b^{2} a^{-1}=a^{2} b^{3}, \\
& x_{k+3}=\prod_{i=3}^{k+2} x_{i}=b^{2} a^{-1}\left(a^{2} b^{3}\right)=a b \\
& x_{k+4}=\prod_{i=4}^{k+3} x_{i}=a^{-1}\left(a^{2} b^{3}\right)(a b)=1 .
\end{aligned}
$$


It follows that $x_{k+j}=1$ for $j(4 \leq j \leq k+1)$. We also have

$$
\begin{aligned}
& x_{2 k+2}=\prod_{i=k+2}^{2 k+1} x_{i}=\left(a^{2} b^{3}\right)(a b)=a, \\
& x_{2 k+3}=\prod_{i=k+3}^{2 k+2} x_{i}=(a b) a=b .
\end{aligned}
$$

It shows that $P_{k}\left(Q_{4 n} ; a, b\right)=2 k+2$.

Let $G=\langle a, b\rangle$ be a finite nonabelian 2-generated group. A 2-step general Fibonacci sequence in the group $G$ is defined by $x_{0}=a, x_{1}=b, x_{i}=x_{i-2}^{m} x_{i-1}^{l}$, for $i \geq 2$, and the integers $m$ and $l$. Now we study this sequence for group $Q_{4 n}$.

Theorem 2.3. Let $m$ and $l$ be integers. If $m \equiv 0(\bmod 2 n)$ or $m \equiv 0(\bmod 4)$, then 2-step general Fibonacci sequence in $Q_{4 n}$ is not simply periodic.

Proof. First we consider the case $m \equiv 0(\bmod 2 n)$. Then the sequence is

$$
x_{0}=a, \quad x_{1}=b, \quad x_{2}=a^{m} b^{l}=b^{l}, \quad x_{3}=b^{m} b^{l^{2}}=b^{m+l^{2}}, \ldots
$$

Obviously, the cycle does not begin again with $a$ and $b$, and hence the sequence is not simply periodic.

Now let $m \equiv 0(\bmod 4)$. Four cases occur.

Case $1(l \equiv 0(\bmod 4))$. Then

$$
\begin{gathered}
x_{0}=a, \quad x_{1}=b, \quad x_{2}=a^{m}, \quad x_{3}=a^{m l}, \\
x_{4}=a^{m^{2}+m l^{2}}, \quad x_{5}=a^{2 m^{2} l+m l^{3}}, \quad x_{6}=a^{m^{3}+3 m^{2} l^{2}+m l^{4}}, \ldots
\end{gathered}
$$

Since the cycle does not begin again with $a$ and $b$, the sequence is not simply periodic.

Case $2(l \equiv 1(\bmod 4))$. Then

$$
x_{0}=a, \quad x_{1}=b, \quad x_{2}=a^{m} b, \quad x_{3}=a^{m} b, \quad x_{4}=a^{m} b, \ldots
$$

Clearly, the sequence is not simply periodic.

Case $3(l \equiv 2(\bmod 4))$. Note that $b^{2}$ is a central element of $Q_{4 n}$. Thus

$$
x_{0}=a, \quad x_{1}=b, \quad x_{2}=a^{m} b^{2}, \quad x_{3}=a^{m l}, \quad x_{4}=a^{m^{2}+m l^{2}}, \quad x_{5}=a^{2 m^{2} l+m l^{3}}, \ldots
$$

Similar to Case 1, the sequence is not simply periodic. 
Case $4(l \equiv 3(\bmod 4))$. So

$$
x_{0}=a, \quad x_{1}=b, \quad x_{2}=a^{m} b^{3}, \quad x_{3}=a^{m} b, \quad x_{4}=a^{m} b^{3}, \quad x_{5}=a^{m} b, \ldots
$$

Thus the sequence is not simply periodic.

Theorem 2.4. Let $n$ be an even integer.

(i) If $m \equiv 1(\bmod 2 n)$ and $l \equiv 0(\bmod 2 n)$, then 2 -step general Fibonacci sequence in $Q_{4 n}$ is simply periodic with period 2.

(ii) If $m \equiv-1(\bmod 2 n)$ and $l \equiv-1(\bmod 2 n)$, then 2 -step general Fibonacci sequence in $Q_{4 n}$ is simply periodic with period 3 .

(iii) If $m \equiv 1(\bmod 2 n)$ and $l \equiv 1(\bmod 2 n)$, then 2 -step general Fibonacci sequence in $Q_{4 n}$ is simply periodic with period 6 .

Proof. (i) Since $n$ is even, $m \equiv 1(\bmod 4)$ and $l \equiv 0(\bmod 4)$. Thus

$$
x_{0}=a, \quad x_{1}=b, \quad x_{2}=a, \quad x_{3}=b, \ldots,
$$

and the period is 2 .

(ii) Since $n$ is even, $m \equiv 3(\bmod 4)$ and $l \equiv 3(\bmod 4)$. Note that $m$ and $l$ are odd integers. So the sequence reduces to

$$
x_{0}=a, \quad x_{1}=b, \quad x_{2}=a^{-1} b^{3}, \quad x_{3}=a, \quad x_{4}=b, \ldots,
$$

and the period is 3 .

(iii) Because $n$ is even, $m \equiv 1(\bmod 4)$ and $l \equiv 1(\bmod 4)$. Furthermore, $m$ and $l$ are odd integers. Then the sequence reduces to

$$
\begin{gathered}
x_{0}=a, \quad x_{1}=b, \quad x_{2}=a b, \quad x_{3}=a^{n-1}, \\
x_{4}=a^{2} b^{3}, \quad x_{5}=a b, \quad x_{6}=a, \quad x_{7}=b, \ldots,
\end{gathered}
$$

and the period is 6 .

Theorem 2.5. Let $n$ be an odd integer.

(i) If $m \equiv 1(\bmod 2 n), m \equiv 1(\bmod 4), l \equiv 0(\bmod 4)$ and $(l, n)=1$, then 2-step general Fibonacci sequence in $Q_{4 n}$ is simply periodic with period $2 n$.

(ii) If $m \equiv 1(\bmod 2 n), m \equiv 3(\bmod 4), l \equiv 0(\bmod 4)$ and $(l, n)=1$, then 2-step general Fibonacci sequence in $Q_{4 n}$ is simply periodic with period $4 n$.

Proof. (i) By induction on $k \geq 0$, we can show that $x_{2 k}=a$ and $x_{2 k+1}=a^{-k l} b$. In particular, the period must be even. Now we have

$$
\left.x_{2 k+1}=b \Longleftrightarrow 2 n|k l \Longleftrightarrow n| k \quad \text { (because }(l, n)=1\right),
$$

and so the period is $2 n$. 
(ii) By induction on $k \geq 0$, it may be shown that

$$
x_{2 k}=a, \quad x_{2 k+1}= \begin{cases}a^{-k l} b & \text { if } k \text { is even, } \\ a^{-k l} b^{3} & \text { if } k \text { is odd. }\end{cases}
$$

Therefore, the period must be even. Now we have

$$
x_{2 k+1}=b \Longleftrightarrow 2 n|k l \Longleftrightarrow 2 n| k \quad \text { (because }(l, n)=1 \text { and } n \text { is odd), }
$$

and thus the period is $4 n$.

Theorem 2.6. Let $n$ and $l$ be odd integers, $m \equiv-1(\bmod 2 n)$ and $(l+1, n)=1$.

(i) If $m \equiv 1(\bmod 4)$, then 2-step general Fibonacci sequence in $Q_{4 n}$ is simply periodic with period $6 n$.

(ii) If $m \equiv 3(\bmod 4)$ and $l \equiv 1(\bmod 4)$, then 2 -step general Fibonacci sequence in $Q_{4 n}$ is simply periodic with period $6 n$.

(iii) If $m \equiv 3(\bmod 4)$ and $l \equiv 3(\bmod 4)$, then 2 -step general Fibonacci sequence in $Q_{4 n}$ is simply periodic with period $3 n$.

Proof. (i) Two cases occur.

Case $1(l \equiv 1(\bmod 4))$. By induction on $k \geq 0$, we can prove that the following relations hold:

$$
\begin{aligned}
x_{6 k} & =a, \\
x_{6 k+1} & =a^{-2 k(l+1)} b, \\
x_{6 k+2} & =a^{-(2 k(l+1)+1)} b, \\
x_{6 k+3} & =a^{n+1}, \\
x_{6 k+4} & =a^{-(n+(2 k+1)(l+1))} b, \\
x_{6 k+5} & =a^{-((2 k+1)(l+1)+1)} b .
\end{aligned}
$$

Consequently, the period must be a multiple of 6 . Now we have

$$
\left.x_{6 k+1}=b \Longleftrightarrow 2 n|2 k(l+1) \Longleftrightarrow n| k \quad \text { (because }(l+1, n)=1\right),
$$

and hence the period is $6 n$.

Case $2(l \equiv 3(\bmod 4))$. The proof is similar to Case 1 except that

$$
\begin{aligned}
& x_{6 k+2}=a^{-(n+2 k(l+1)+1)} b, \\
& x_{6 k+4}=a^{-(2 k+1)(l+1)} b .
\end{aligned}
$$


(ii) It is easily shown that the following relations hold for every $k \geq 0$ :

$$
\begin{aligned}
& x_{3 k}=a, \quad x_{6 k}=a, \quad x_{6 k+1}=a^{-2 k(l+1)} b, \quad x_{6 k+2}=a^{-(2 k(l+1)+1)} b, \\
& x_{6 k+3}=a, \quad x_{6 k+4}=a^{-(n+(2 k+1)(l+1))} b, \quad x_{6 k+5}=a^{-(n+1+(2 k+1)(l+1))} b .
\end{aligned}
$$

Particularly, the period must be a multiple of 3 . Since $n+(2 k+1)(l+1)$ is odd, then it is not a multiple of $2 n$. Thus $x_{6 k+4} \neq b$. Further,

$$
\left.x_{6 k+1}=b \Longleftrightarrow 2 n|2 k(l+1) \Longleftrightarrow n| k \quad \text { (because }(l+1, n)=1\right),
$$

and hence the period is $6 n$.

(iii) By induction on $k \geq 0$, we can prove the following relations:

$$
\begin{aligned}
x_{3 k} & =a, \\
x_{3 k+1} & =a^{-k(l+1)} b, \\
x_{3 k+2} & =a^{-(n+1+k(l+1))} b .
\end{aligned}
$$

Therefore, the period must be a multiple of 3. Now we have

$$
\left.x_{3 k+1}=b \Longleftrightarrow 2 n|k(l+1) \Longleftrightarrow n| k \quad \text { (because }(l+1, n)=1\right),
$$

and so the period is $3 n$.

\section{Acknowledgments}

The authors would like to express their appreciations to the referees for the valuable and constructive comments regarding the presentation of this paper.

\section{References}

[1] D. D. Wall, "Fibonacci series modulo m," The American Mathematical Monthly, vol. 67, pp. 525-532, 1960.

[2] H. J. Wilcox, "Fibonacci sequences of period $n$ in groups," The Fibonacci Quarterly, vol. 24, no. 4, pp. 356-361, 1986.

[3] C. M. Campbell, H. Doostie, and E. F. Robertson, "Fibonacci length of generating pairs in groups," in Applications of Fibonacci Numbers, vol. 3, pp. 27-35, Kluwer Academic Publishers, Dordrecht, The Netherlands, 1990.

[4] S. W. Knox, "Fibonacci sequences in finite groups," The Fibonacci Quarterly, vol. 30, no. 2, pp. 116-120, 1992.

[5] H. Aydin and G. C. Smith, "Finite p-quotients of some cyclically presented groups," Journal of the London Mathematical Society, vol. 49, no. 1, pp. 83-92, 1994.

[6] R. Dikici and G. C. Smith, "Fibonacci sequences in finite nilpotent groups," Turkish Journal of Mathematics, vol. 21, no. 2, pp. 133-142, 1997.

[7] R. Dikici and G. C. Smith, "Recurrences in finite groups," Turkish Journal of Mathematics, vol. 19, no. 3, pp. 321-329, 1995. 
[8] H. Aydin and R. Dikici, "General Fibonacci sequences in finite groups," The Fibonacci Quarterly, vol. 36, no. 3, pp. 216-221, 1998.

[9] E. Karaduman and U. Yavuz, "On the period of Fibonacci sequences in nilpotent groups," Applied Mathematics and Computation, vol. 142, no. 2-3, pp. 321-332, 2003.

[10] E. Karaduman and H. Aydin, "General 2-step Fibonacci sequences in nilpotent groups of exponent $p$ and nilpotency class 4," Applied Mathematics and Computation, vol. 141, no. 2-3, pp. 491-497, 2003.

[11] E. Karaduman and H. Aydin, "On Fibonacci sequences in nilpotent groups," Mathematica Balkanica, vol. 17, no. 3-4, pp. 207-214, 2003.

[12] R. Dikici and A. Işik, "Fibonacci sequences in modular groups," Institute of Mathematics E Computer Sciences, vol. 10, no. 3, pp. 187-189, 1997.

[13] R. Dikici and E. Özkan, "General recurrences in modular groups," Journal of the Faculty of Science, vol. 21, no. 2, pp. 93-101, 1998.

[14] H. Doostie and R. Golamie, "Computing on the Fibonacci lengths of finite groups," International Journal of Applied Mathematics, vol. 4, no. 2, pp. 149-156, 2000.

[15] H. Doostie and M. Maghasedi, "Fibonacci length of direct products of groups," Vietnam Journal of Mathematics, vol. 33, no. 2, pp. 189-197, 2005.

[16] H. Doostie and C. M. Campbell, "Fibonacci length of automorphism groups involving Tribonacci numbers," Vietnam Journal of Mathematics, vol. 28, no. 1, pp. 57-65, 2000.

[17] C. M. Campbell and P. P. Campbell, "The Fibonacci length of certain centro-polyhedral groups," Journal of Applied Mathematics \& Computing, vol. 19, no. 1-2, pp. 231-240, 2005.

[18] C. M. Campbell, P. P. Campbell, H. Doostie, and E. F. Robertson, "Fibonacci lengths for certain metacyclic groups," Algebra Colloquium, vol. 11, no. 2, pp. 215-222, 2004.

[19] H. Doostie and M. Hashemi, "Fibonacci lengths involving the Wall number $k(n)$," Journal of Applied Mathematics \& Computing, vol. 20, no. 1-2, pp. 171-180, 2006.

[20] E. Karaduman and H. Aydin, "On the periods of 2-step general Fibonacci sequences in dihedral groups," Matematichki Vesnik, vol. 58, no. 1-2, pp. 47-56, 2006. 


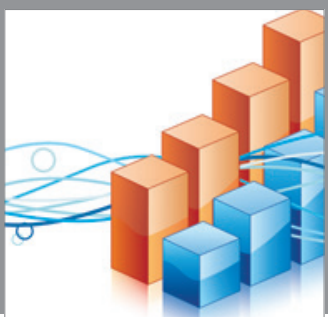

Advances in

Operations Research

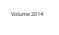

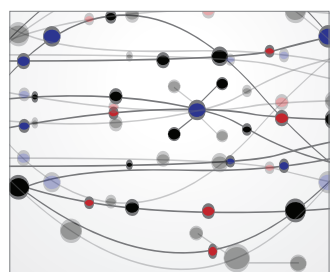

\section{The Scientific} World Journal
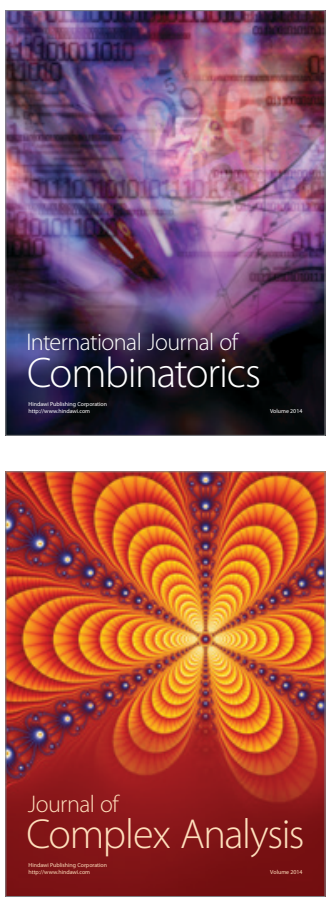

International Journal of

Mathematics and

Mathematical

Sciences
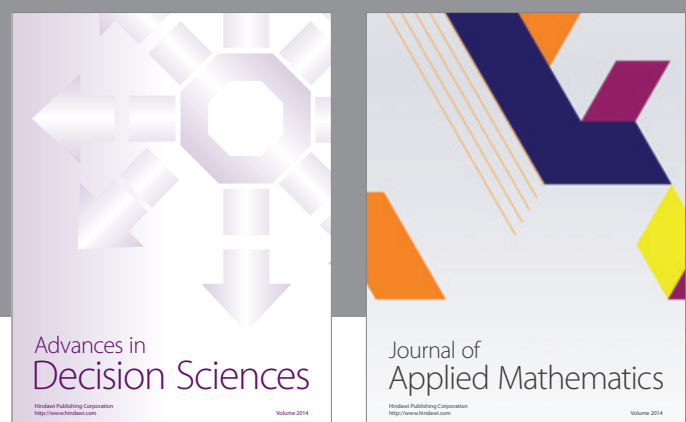

Journal of

Applied Mathematics
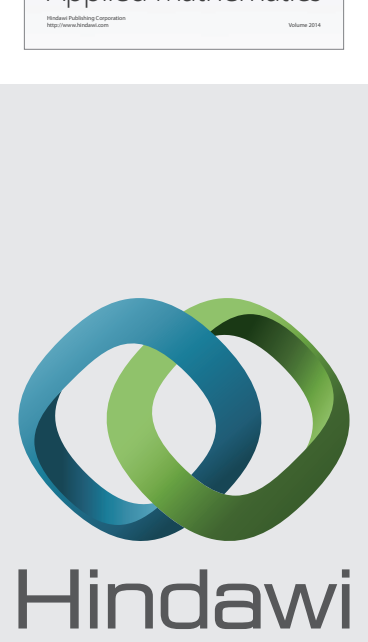

Submit your manuscripts at http://www.hindawi.com
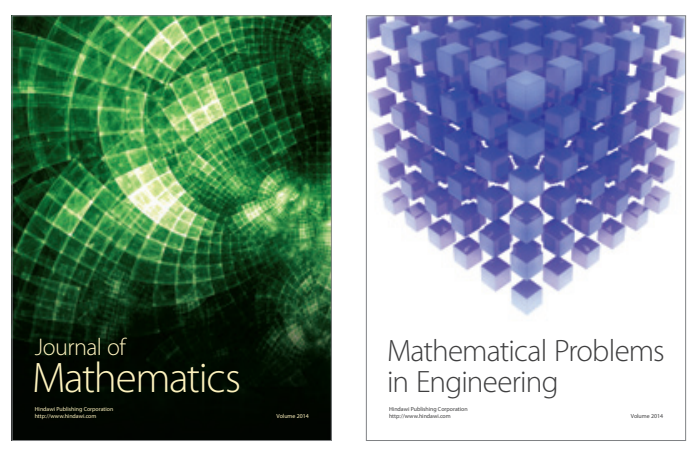

Mathematical Problems in Engineering
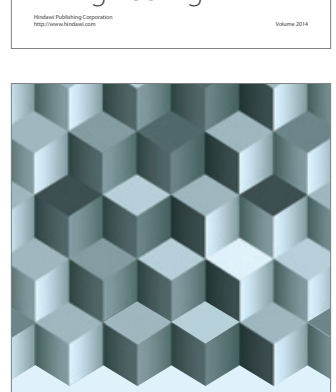

Journal of

Function Spaces
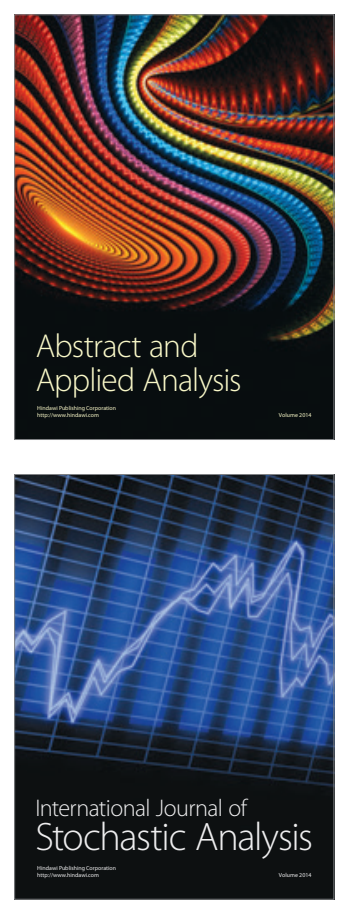

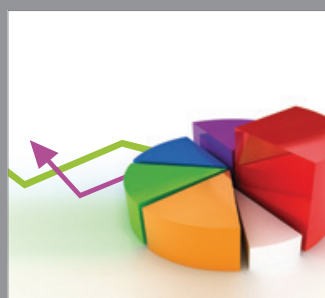

ournal of

Probability and Statistics

Promensencen
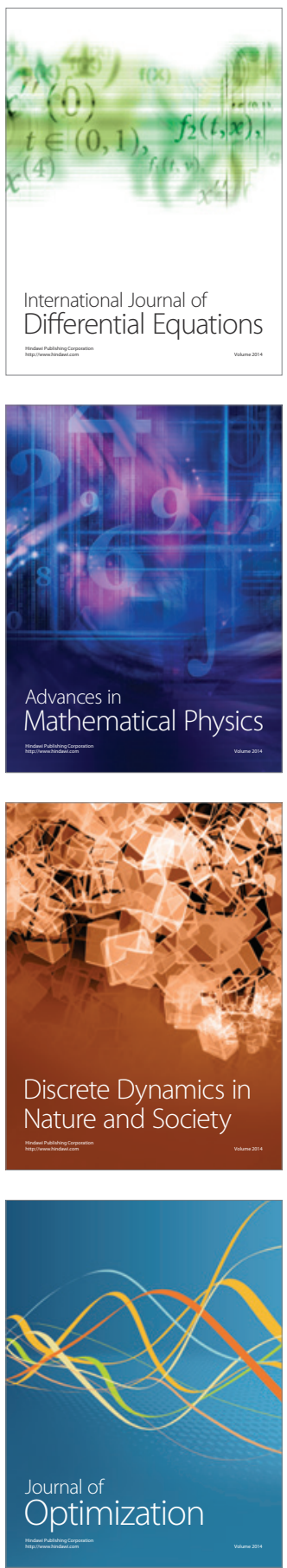